\title{
On the Static Properties of Baryons in the Skyrme Model
}

\author{
O. L. Battistel \\ Centro Universitário Franciscano, Rua dos Andradas, 1614, 97010-032, Santa Maria, RS, Brasil
}

Received on 9 September, 2003

\begin{abstract}
We use a modified version of the Skyrme model in which the stabilizing fourth-order term is separated in two parts with different parameters that are introduced in the Lagrangian and could vary independently. The predictions for the static properties of baryons are calculated and results are compared with the respective experimental values and those of other authors. We show that it possible to improve in an expressive way the results for the set of the important quantities at the same time.
\end{abstract}

\section{Introduction}

In the early 1960's T.H.R. Skyrme published a pioneering series of work in which he developed the idea that nucleons are solitons in a field theory of mesons. He proposed to use the non-linear $\sigma$-model to describe the light pseudo-scalar mesons in which the non-strange baryons emerge as stable field configurations with a non-trivial geometrical structure. Skyrme's soliton was a hedgehog configuration of the meson field with a conserved topological charge or winding number. He made the fundamental assumption that this winding number was in fact identical to baryon number. He studied the properties of these solitons, and obtained estimates for the meson field in terms of the parameters of his model.

Twenty years later, with the advent of concepts such as chiral symmetry and QCD, Witten [1] and others demonstrated the link of the Skyrme model to QCD by investigating the underlying symmetries and connecting the anomalies of QCD to the Wess-Zumino term [2], which renewed the interest in this model. General reviews of the Skyrme model have been written by Holzwarth and Schwesinger [3], Zahed and Brown [4] and Meissner [5], among others.

In spite of the non-linear $\sigma$-model has provided a rather economical framework for discussing low-energy phenomenology, his finite field configurations are not energetically stable in 3-space by Derrick's theorem [6], i.e., this solitons are unstable against scale transformations. To avoid this collapse, Skyrme added by hand a quartic term in the currents $\left(\mathcal{L}_{4}\right)$ to the Lagrangian of non-linear $\sigma$-model $\left(\mathcal{L}_{2}+\mathcal{L}_{m \pi}\right)$, with a dimensionless parameter $(e)$ that characterizes the size of the finite energy configurations. This term can be understood as a higher-order correction to the non-linear $\sigma$-model when cast in the general framework of an effective chiral description as advocated by Weinberg [7]. The presence of higher order terms is important because there are problems with stability of the classical solution when such terms are included. The question of non-Skyrme four-derivative terms appears in the interaction between two nucleons in the Skyrme model. In the Paris potential the attractive scalar interaction is treated by $\pi N$ and $\pi \pi$ disper- sion relations. This procedure leads to a term in the Skyrme Lagrangian additional to Skyrme term. The numerical analysis of the Paris group [8] shows that the skyrmion becomes unstable when the strength of this term increases.

\section{Our Analysis}

We studied the predictions of the model in various approaches. Our analysis [9] includes different ways to determine de parameters in the original Lagrangian $\mathcal{L}_{2}+\mathcal{L}_{4}+$ $\mathcal{L}_{m \pi}$, stabilization via $\omega$ meson, $\gamma \omega_{\mu} B^{\mu}$, and with the introduction of sixth-order term $\beta B_{\mu} B^{\mu}$, where $B^{\mu}$ is the anomalous baryon current. Table 1 resumes some results obtained for the static properties of baryons in this calculations.

In this table $\left\langle r_{E}^{2}>_{B}\right.$ and $\left\langle r_{E}^{2}>_{V}\right.$ are the isoscalar and isovector mean square electric radii, $<r_{M}^{2}>_{B}$ and $<r_{M_{V}}^{2}>_{V}$ are the mean square magnetic radii and $\mu^{B}$ and $\mu^{V}$ are the isoscalar and isovector magnetic moments. These quantities are simply related $[3,4,5]$ to the proton and neutron electric and magnetic radii $<r_{E, M}^{2}>_{p, n}$ and respective magnetic moments $\mu_{p, n}$. We also include the axialvector coupling constant $g_{A}$.

On the overall the results are satisfactory, but we verify that in the conventional and other variations the quantities cannot be described together with high degree of accuracy. We show that exist structural relations and numerical correlations among them and this lead us to conclude that the model could be sub parameterized. This implies that an improvement in any of them results in worse values for others and that it is impossible by simple variation of the parameters obtain correct values for all quantities.

As a remedy of this inherent link among quantities in the Skyrme model, in this analysis we proposed to modify the Lagrangian of the model separating the stabilizing fourthorder term in two parts proportional to parameters $e_{1}$ and $e_{2}$, such that the Lagrangian density for the static soliton acquires the form 
TABLE I. Static properties of baryons calculated in conventional Skyrme model (conv.1 and conv.2), with vector $\omega$ and with sixth-order term $B_{\mu} B^{\mu}$.

\begin{tabular}{|c|c|c|c|c|c|}
\hline Quantity & Conv.1 & Conv. 2 & $\omega$ & $B_{\mu} B^{\mu}$ & Exp. \\
\hline$f_{\pi}(\mathrm{MeV})$ & 54.0 & 93.0 & 62.0 & 65.1 & 93.0 \\
\hline parameter & $\mathrm{e}=4.84$ & $\mathrm{e}=4.0$ & $\gamma=15.64$ & $\beta=0.019$ & - \\
\hline$<r_{E}^{2}>_{B}^{\frac{1}{2}}(\mathrm{fm})$ & 0.6833 & 0.5083 & 0.7482 & 0.7571 & 0.72 \\
\hline$<r_{E}^{2}>_{V}^{\frac{1}{2}}(\mathrm{fm})$ & 1.0405 & 0.8665 & 1.0851 & 1.0396 & 0.88 \\
\hline$<r_{M}^{2}>_{B}^{\frac{1}{2}}(\mathrm{fm})$ & 0.7402 & 0.5631 & 0.7371 & 0.7085 & 0.82 \\
\hline$<r_{M}^{2}>_{V}^{\frac{1}{2}}(\mathrm{fm})$ & 0.8059 & 0.6712 & 0.8405 & 0.8053 & 0.80 \\
\hline$\mu^{B}$ (magn.) & 0.3655 & 0.1726 & 0.4295 & 0.4029 & 0.44 \\
\hline$\mu^{V}$ (magn.) & 1.6067 & 1.8824 & 1.6446 & 1.6072 & 2.35 \\
\hline$\mu_{p}$ (magn.) & 1.9722 & 2.0551 & 2.0742 & 2.0102 & 2.79 \\
\hline$\mu_{n}$ (magn.) & -1.2412 & -1.7097 & -1.2151 & -1.2043 & -1.91 \\
\hline$<r_{E}^{2}>_{p}^{\frac{1}{2}}(\mathrm{fm})$ & 0.8821 & 0.7124 & 0.9320 & 0.8932 & $0.86 \pm 0.01$ \\
\hline$<r_{E}^{2}>_{n}\left(f m^{2}\right)$ & -0.3079 & -0.2462 & -0.3088 & -0.2830 & $-0.119 \pm 0.004$ \\
\hline$<r_{M}^{2}>_{p}^{\frac{1}{2}}(\mathrm{fm})$ & 0.7942 & 0.6628 & 0.9432 & 0.7869 & $0.86 \pm 0.06$ \\
\hline$<r_{M}^{2}>_{n}^{\frac{1}{2}}(\mathrm{fm})$ & 0.8243 & 0.6811 & 0.6274 & 0.8352 & $0.88 \pm 0.07$ \\
\hline$g_{A}$ & 0.65 & 1.01 & - & - & $1.25 \pm 0.01$ \\
\hline
\end{tabular}

$$
\mathcal{L}=-\frac{f_{\pi}^{2}}{2}\left[F^{\prime 2}+\frac{2 \sin ^{2} F}{r^{2}}\right]-\frac{1}{e_{1}^{2}} \frac{\sin ^{2} F}{r^{2}} F^{\prime 2}-\frac{1}{2 e_{2}^{2}} \frac{\sin ^{4} F}{r^{4}}-m_{\pi}^{2} f_{\pi}^{2}(1-\cos F)
$$

where $F$ is the chiral angle or profile function. The aim of this procedure is just verify if the introduction of new free parameter could decouple some results and allow the adjust of the values obtained for the set of the quantities of interest.
Using the conventional method, we utilize the Lagrangian (1) and perform the calculations in the model to obtain the desired quantities. Some of them are listed in table 2 .

TABLE II. Static properties of baryons calculated in a modified version of Skyrme model.

\begin{tabular}{|l|c|c|c|c|}
\hline \multicolumn{1}{|c|}{ Quantity } & $e_{1}=3,9 e_{2}=3,4$ & $e_{1}=3,9 e_{2}=3,5$ & $e_{1}=3,9 e_{2}=3,6$ & exp. \\
\hline$f_{\pi}(\mathrm{MeV})$ & 93,0 & 93,0 & 93,0 & 93,0 \\
\hline$<r_{E}^{2}>_{B}^{\frac{1}{2}}(\mathrm{fm})$ & 0,5366 & 0,5332 & 0,5297 & 0,72 \\
\hline$<r_{E}^{2}>_{V}^{\frac{1}{2}}(\mathrm{fm})$ & 0,8772 & 0,8777 & 0,8780 & 0,88 \\
\hline$\left.<r_{M}^{2}\right\rangle_{B}^{\frac{1}{2}}(\mathrm{fm})$ & 0,5843 & 0,5825 & 0,5806 & 0,82 \\
\hline$<r_{M}^{2}>_{V}^{\frac{1}{2}}(\mathrm{fm})$ & 0,6794 & 0,6798 & 0,6801 & 0,80 \\
\hline$\mu^{B}$ (magn.) & 0,1615 & 0,1631 & 0,1646 & 0,44 \\
\hline$\mu^{V}$ (magn.) & 2,2428 & 2,1924 & 2,1439 & 2,35 \\
\hline$\mu_{p}$ (magn.) & 2,4043 & 2,3556 & 2,3086 & 2,79 \\
\hline$\mu_{n}$ (magn.) & $-2,0813$ & $-2,0293$ & $-1,9793$ & $-1,91$ \\
\hline$<r_{E}^{2}>_{p}^{\frac{1}{2}}(\mathrm{fm})$ & 0.7271 & 0,7261 & 0,7251 & $0,86 \pm 0,01$ \\
\hline$<r_{E}^{2}>_{n}\left(f m^{2}\right)$ & $-0,2407$ & $-0,2430$ & $-0,2451$ & $-0,119 \pm 0,004$ \\
\hline$<r_{M}^{2}>_{p}^{\frac{1}{2}}(\mathrm{fm})$ & 0,6735 & 0,6735 & 0,6735 & $0,86 \pm 0,06$ \\
\hline$<r_{M}^{2}>_{n}^{\frac{1}{2}}(\mathrm{fm})$ & 0,6863 & 0,6870 & 0,6877 & $0,88 \pm 0,07$ \\
\hline$g_{A}$ & 1,2865 & 1,2347 & 1,1830 & $1,25 \pm 0,01$ \\
\hline
\end{tabular}


In tables 1 and 2 the results for the static properties of the baryons predicted in different versions of the model are shown. It is interesting to observe the comportment of some results like $<r_{E}^{2}>_{n}$ and $g_{A}$. While the first seems to be insensible, the second presents large variations in different formulations.

\section{Conclusion}

We use an alternative form of the Lagrangian of the Skyrme model to calculate the static properties of the baryons. This study is set out with a simple purpose: verify in what sense the introduction of more parameters in the conventional form could alter an apparent internal inconsistency of the model in what the quantities presents some link between them. We verify that the value calculated for $\left\langle r_{E}^{2}\right\rangle_{n}$ stay almost constant in any version utilized. It seems that this is an artifact of the model. In other cases we verify that in this approach it is possible to produce better results for the set of quantities studied. Some results that are problematic in the calculations such that presented by Adkins et al [10] and by Holzwarth et al [11] could be sensitively improved. This is the case for $\mu_{p}, \mu_{n}$ and $g_{A}$. In other hand the fact that the quantities have been well predicted maintaining the remain around their traditional values indicates that this procedure permits to decouple the results as we expect. In the specific case of pion decay constant $f_{\pi}$ and axial-vector coupling constant $g_{A}$, in any version of the model discussed in the literature their values have been not well predicted at same time. In our analysis the introduction of two parameters permits de desired flexibility of the model and these values are correctly obtained.

\section{References}

[1] E. Witten, Nucl. Phys. B233, 422, 433 (1983).

[2] J. Wess and B. Zumino, Phys. Lett. B37, 95 (1971).

[3] G. Holzwarth and B. Schwesinger, Rep. Prog. Phys. 49, 825 (1986).

[4] I. Zahed and G.E. Brown, Phys. Rep. 142, 1 (1986).

[5] U. Meissner, Phys. Rep. 161, 213 (1988).

[6] G.H. Derrick, J. Math. Phys. 51252 (1964).

[7] S. Weinberg, Physica 96, 325 (1979).

[8] M. Lacombe, B. Loiseau, R. Vinh Mau, and W.N. Cottingham, Phys. Lett. B 169, 121 (1986).

[9] O. L. Battistel, PhD Thesis, USP (1994);

[10] G.S. Adkins, C.R. Nappi, and E. Witten, Nucl. Phys. B228, 552 (1983).

[11] G. Holzwarth, G. Pari, and B.K. Jennings, Nucl. Phys. A515, 665 (1990). 